\title{
Predictive Haemodynamics in a One-Dimensional Human Carotid Artery Bifurcation. Part II: Application to Graft Design
}

\author{
Vijaya B. Kolachalama, Member, IEEE, Neil W. Bressloff*, Prasanth B. Nair, and Clifford P. Shearman
}

\begin{abstract}
A Bayesian surrogate modeling technique is proposed that may be able to predict an optimal bypass graft configuration for patients suffering with stenosis in the internal carotid artery (ICA). At the outset, this statistical technique is considered as a means for identifying key geometric parameters influencing haemodynamics in the human carotid bifurcation. This methodology uses a design of experiments (DoE) technique to generate candidate geometries for flow analysis. A pulsatile one-dimensional Navier-Stokes solver incorporating fluid-wall interactions for a Newtonian fluid which predicts pressure and flow in the carotid bifurcation (comprising a stenosed segment in the internal carotid artery) is used for the numerical simulations. Two metrics, pressure variation factor (PVF) and maximum pressure $\left(p_{m}^{*}\right)$ are employed to directly compare the global and local effects, respectively, of variations in the geometry. The values of PVF and $p_{m}^{*}$ are then used to construct two Bayesian surrogate models. These models are statistically analyzed to visualize how each geometric parameter influences PVF and $p_{m}^{*}$. Percentage of stenosis is found to influence these pressure based metrics more than any other geometric parameter. Later, we identify bypass grafts with optimal geometric and material properties which have low values of PVF on five test cases with $70 \%, 75 \%, 80 \%, 85 \%$, and $90 \%$ stenosis in the ICA, respectively.
\end{abstract}

Index Terms-1-D blood flow, Bayesian modeling, carotid artery, graft design, parametric studies, statistical analysis.

\section{INTRODUCTION}

$\mathbf{T}$ HE CAROTID artery bifurcation is a common site for arterial disease in the human body. One of the most frequently accomplished interventional procedures in the carotids is endarterectomy [1], and an increasingly popular alternative involves angioplasty followed by stenting [2]. However, for some patients, bypass grafting may provide the preferred means for

Manuscript received January 4, 2007; revised September 4, 2007. Asterisk indicates corresponding author.

V. B. Kolachalama is with the Biomedical Engineering Center, Harvard-MIT Division of Health Sciences and Technology, Cambridge, MA 02139 USA (e-mail : vbk@mit.edu).

$* \mathrm{~N}$. W. Bressloff is with the Computational Engineering and Design Center, School of Engineering Sciences, University of Southampton, Southampton SO17 1BJ, U.K. (e-mail: nwb@soton.ac.uk).

P. B. Nair is with the Computational Engineering and Design Center, School of Engineering Sciences, University of Southampton, Southampton SO17 1BJ,

U.K. (e-mail: pbn@ soton.ac.uk).

C. P. Shearman is with the Department of Vascular Surgery, Southampton General Hospital, Southampton SO16 6YD, U.K. (e-mail: c.p.shearman@soton.ac.uk).

Color versions of one or more of the figures in this paper are available online at http://ieeexplore.ieee.org.

Digital Object Identifier 10.1109/TBME.2007.912398

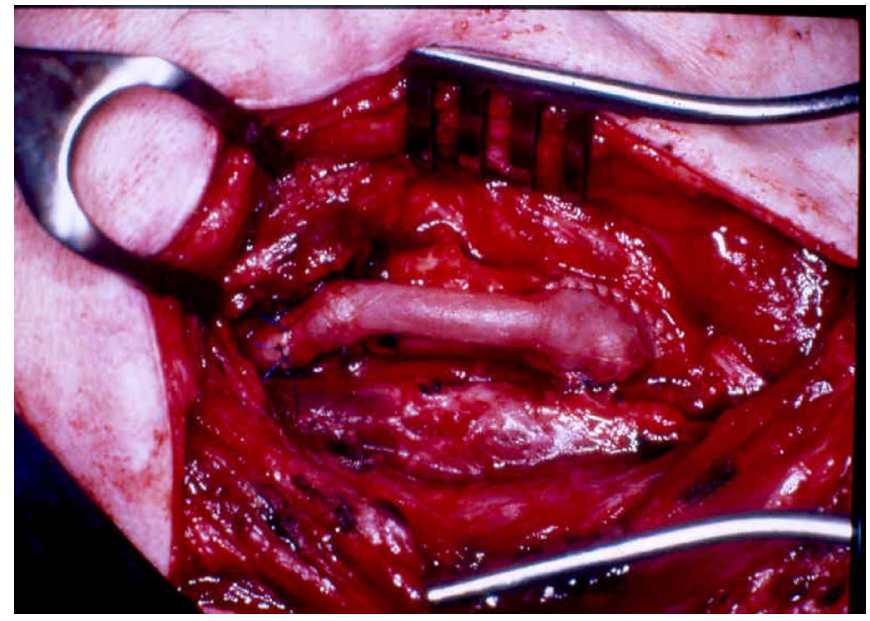

Fig. 1. Operative picture of saphenous vein bypass graft from the common carotid artery (CCA) (on right of picture) to the internal carotid artery (ICA) (on left). The anastomosis to the CCA is end to side and the anastomosis to the ICA is end-to-end. The patient had atherosclerotic disease at the origin of the ICA.

carotid artery reconstruction, especially when technical difficulties are associated with endarterectomy or angioplasty [3]. Such situations can occur for patients having complex carotid geometries with high tortuosity and in cases where the extent of stenosis is large. In addition, extracranial-intracranial arterial bypass surgery has been found to be promisingly efficacious in the treatment of haemodynamic impairment due to unilateral internal carotid artery occlusion [4]. Typically, when grafting, large autogenous veins (e.g., the autologous saphenous vein) are taken from the patient's leg or prosthetic veins are harvested (cf. Fig. 1). The purpose of these vascular conduits is to shunt the blood flow around a blockage. Although all of the above techniques are commonly used and clinically approved, significant postsurgical problems can occur inside the native vessel often involving restenosis (neointimal hyperplasia). In the case of grafting, graft failure may occur due to the compliance mismatch at the junction between the native vessel and the graft [5]. In addition, there is some evidence to suggest that the geometry of the arterial bypass can significantly influence the postoperative haemodynamic environment [6], [7]. Hence, selection of an optimal shape and a suitable material for a bypass graft could play a critical role in inhibiting mechanical failure. With this possibility in mind, a novel technique for designing a patient specific bypass graft is presented, which may be able to improve the treatment of haemodynamic compromise. 
The present work extends the methodology developed in [8], where the use of predictive tools highlighted the importance of a parametric study and its subsequent potential application to predict the benefit of stenting in a diseased patient. As an alternative to the approach in [8], statistical techniques have also been used in a preliminary study to understand the influence of inter/intra individual geometric differences on flow behavior [9], [10]. The application of these techniques developed for optimizing graft design are discussed here.

In this paper, a deterministic numerical model which can predict flow and pressure in the human carotid bifurcation using a time dependent one-dimensional Navier-Stokes solver for a Newtonian fluid is used. Furthermore, a Bayesian surrogate modeling technique is employed to conduct a statistical analysis to rank the key geometric parameters which influence pressure variation factor (PVF) and maximum pressure $\left(p_{m}^{*}\right)$ in the carotid artery. Originally introduced in [8], PVF is defined as

$$
\mathrm{PVF}=\frac{\int_{t}^{t+j T} \int_{0}^{L}\left(p_{s}-p\right)^{2} d x d t}{\int_{t}^{t+j T} \int_{0}^{L} p^{2} d x d t}
$$

where $p_{s}(x, t+j T)=p_{s}(x, t)$ and $p(x, t+j T)=p(x, t)$ (for $j=1,2,3, \ldots$ ) are the instantaneous pressure in the presence of a stenosis and the instantaneous pressure when there is no stenosis, respectively. This nondimensional metric quantifies the extent of deviation of pressure from ideal behavior in the entire cross section of a single arterial vessel. In addition, $p_{m}^{*}$ is defined as

$$
p_{m}^{*}=\frac{\operatorname{Max}\left[p_{s}(x, t)\right]}{\operatorname{Max}[p(x, t)]} .
$$

This metric gives an estimate of local pressure increment. The choice of PVF and $p_{m}^{*}$ as target variables is based on the intention to provide a measure of global and local variation due to the presence of the graft, such that a graft is sought that minimizes these variations. While variations in pressure have been considered here, metrics could have been similarly employed based on the flow rate.

A parameterized model of the one-dimensional human carotid artery bifurcation is used to automatically construct a range of different geometries. The basic idea is to use a design of experiments (DoE) technique to generate a set of candidate geometries (at which the flow solver is run) that not only comprise significant variations in the levels of stenosis in the internal carotid artery (ICA), but also have shape differences at other locations in the bifurcation. The data generated from these runs is then used to construct a Bayesian interpolant which approximates the objective as a function of the geometric variables. This model is subsequently employed as a computationally cheap surrogate for further statistical analysis. More specifically, the degree of influence of each geometric parameter on PVF and $p_{m}^{*}$ is estimated. Later, five test cases with different levels of stenosis in the ICA are considered and the application of the Bayesian model to determine a graft configuration that has a low value of PVF in the carotid bifurcation is discussed. In what follows, we briefly present the theoretical aspects of Bayesian surrogate modeling. Finally, key results are presented and discussed.

\section{BAYESIAN MODELING}

This section presents the theory of Bayesian Gaussian process interpolation/regression. Consider a deterministic computational fluid dynamics (CFD) code which takes as input the vector $\mathbf{x} \in \mathbb{R}^{p}$ and returns a scalar output $y(\mathbf{x})$. Further, for a given set of $l$ input vectors $\mathbf{X}=\left\{\mathbf{x}^{1}, \mathbf{x}^{2}, \ldots, \mathbf{x}^{l}\right\} \in \mathbb{R}^{p \times l}$, the corresponding output values $\mathbf{y}=\left\{y^{1}, y^{2}, \ldots, y^{l}\right\} \in \mathbb{R}^{l}$ are assumed to be available. This training data may typically be obtained in practice by applying a DoE technique [11] to decide candidate geometries at which the flow solver should be run. Using this training data, the approximation problem involves the prediction of the output $y(\mathbf{x})$ given a new design point $\mathbf{x}$.

The metamodel used in Bayesian interpolation can be compactly written as

$$
Y(\mathbf{x})=\beta+Z(\mathbf{x})
$$

where $\beta$ is an unknown hyperparameter to be estimated from the data and $Z(\mathbf{x})$ is a Gaussian stochastic process with zero-mean and covariance

$$
\operatorname{Cov}\left(\mathrm{Z}(\mathbf{x}), \mathrm{Z}\left(\mathbf{x}^{\prime}\right)\right)=\sigma_{z}^{2} \mathrm{R}\left(\mathbf{x}, \mathbf{x}^{\prime}\right)
$$

where $R(. .$.$) is a correlation function that can be tuned to the$ data and $\sigma_{z}^{2}$ is the so called process variance. A commonly used choice of correlation function is the stationary family which obeys the product correlation rule [12]

$$
R\left(\mathbf{x}^{1}, \mathbf{x}^{2}\right)=\prod_{j=1}^{p} \exp \left(-\theta_{j}\left|\mathbf{x}_{j}^{1}-\mathbf{x}_{j}^{2}\right|^{p_{j}}\right)
$$

where $\theta_{j} \geq 0$ and $0<p_{j} \leq 2$ are the hyperparameters. In the present study, we have chosen $p_{j}=2$ to reflect the belief that the underlying function being modeled is smooth and infinitely differentiable. The hyperparameters $\theta_{j}$ which control the nonlinearity of the metamodel are estimated from the data. For example, small values of $\theta_{j}$ indicate that the output is a smooth function of the $j$ th variable, and high values indicate highly nonlinear behavior. It is also possible to tune the parameters $p_{j}$ to the data which allows for the possibility of modeling functions which may be discontinuous. In theory, the choice of an optimal covariance function is data-dependent. However, in practice it has been found that the parameterized covariance function in (4) offers sufficient flexibility for modeling smooth and highly nonlinear functions [13].

Assuming Gaussian prior over functions, and by applying Bayes' theorem, it can be shown that the posterior distribution is Gaussian [14], i.e., $Y(\mathbf{x}) \mid \mathbf{y} \sim \mathcal{N}\left(\widehat{Y}(\mathbf{x}), \sigma_{z}^{2} C\left(\mathbf{x}, \mathbf{x}^{\prime}\right)\right)$. The posterior mean and covariance can be computed as

$$
\widehat{Y}(\mathbf{x})=\beta+\mathbf{r}(\mathbf{x})^{T} \mathbf{R}^{-1}(\mathbf{y}-\mathbf{1} \widehat{\beta})
$$

and

$$
C\left(\mathbf{x}, \mathbf{x}^{\prime}\right)=\sigma_{z}^{2}\left(R\left(\mathbf{x}, \mathbf{x}^{\prime}\right)-\mathbf{r}(\mathbf{x})^{T} \mathbf{R}^{-1} \mathbf{r}\left(\mathbf{x}^{\prime}\right)\right)
$$

where $\mathbf{R} \in \mathbb{R}^{l \times l}$ is the correlation matrix computed using the training points; the $i j$ th element of this matrix is computed as 
$\mathbf{R}_{i j}=R\left(\mathbf{x}^{i}, \mathbf{x}^{j}\right) \cdot \mathbf{r}(\mathbf{x})=\left\{R\left(\mathbf{x}, \mathbf{x}^{1}\right) R\left(\mathbf{x}, \mathbf{x}^{2}\right) \ldots R\left(\mathbf{x}, \mathbf{x}^{l}\right)\right\} \in$ $\mathbb{R}^{l}$ is the correlation between the new point $\mathbf{x}$ and the training points and $\mathbf{1}=\{1,1, \ldots, 1\} \in \mathbb{R}^{l}$. It can be observed from (6) and (7) that the Bayesian inferencing approach leads to an approximation of the CFD code as a multidimensional Gaussian random field. The posterior covariance given in (7) can be interpreted as an estimate of the uncertainty involved in making predictions at a new point $\mathbf{x}$. Note that this uncertainty arises from the fact that only a finite set of points are used to construct the surrogate model.

In practice, for the sake of computational efficiency, we compute the Cholesky decomposition of $\mathbf{R}$. This allows the posterior mean to be computed using a vector-vector product, i.e., $\widehat{Y}(\mathbf{x})=\beta+\mathbf{r}(\mathbf{x})^{T} \mathbf{w}$, where $\mathbf{w}=\mathbf{R}^{-1}(\mathbf{y}-\mathbf{1} \widehat{\beta})$. However, the computation of the variance (or error bar) of the posterior process (i.e., $C(\mathbf{x}, \mathbf{x}))$ requires a forward and back substitution.

\section{A. Maximum-Likelihood Estimation}

The hyperparameters $\boldsymbol{\theta}=\left\{\theta_{j}\right\}, j=1,2, \ldots, p, \beta$ and $\sigma_{z}^{2}$ which arise in the correlation function defined in (4) can be computed using a maximum-likelihood estimation (MLE) procedure. For the case of Gaussian process interpolation, the likelihood function is

$$
(2 \pi)^{-l / 2}\left(\sigma_{z}^{2}\right)^{-l / 2}|\mathbf{R}|^{-1 / 2} \exp \left[-\frac{1}{2 \sigma_{z}^{2}}(\mathbf{y}-\mathbf{1} \beta)^{T} \mathbf{R}^{-1}(\mathbf{y}-\mathbf{1} \beta)\right] .
$$

Hence, the negative log-likelihood function to be minimized becomes

$$
\begin{aligned}
& L\left(\boldsymbol{\theta}, \beta, \sigma_{z}^{2}\right)= \\
& -\frac{1}{2}\left[l \ln (2 \pi)+l \ln \sigma_{z}^{2}+\ln |\mathbf{R}|+\frac{1}{\sigma_{z}^{2}}(\mathbf{y}-\mathbf{1} \beta)^{T} \mathbf{R}^{-1}(\mathbf{y}-\mathbf{1} \beta)\right] .
\end{aligned}
$$

Given the maximum-likelihood estimate of $\boldsymbol{\theta}$, the parameters $\beta$ and $\sigma_{z}^{2}$ can be estimated as

$$
\begin{aligned}
\widehat{\beta} & =\left(\mathbf{1}^{T} \mathbf{R}^{-1} \mathbf{1}\right)^{-1} \mathbf{1}^{T} \mathbf{R}^{-1} \mathbf{y} \\
\widehat{\sigma_{z}^{2}} & =\frac{1}{l}(\mathbf{y}-\mathbf{1} \beta)^{T} \mathbf{R}^{-1}(\mathbf{y}-\mathbf{1} \beta) .
\end{aligned}
$$

By substituting the value of $\widehat{\sigma_{z}^{2}}$ from (11) in (9) and by removing the term $l \ln (2 \pi)$, the negative $\log$-likelihood function becomes

$$
L\left(\boldsymbol{\theta}, \beta, \sigma_{z}^{2}\right)=-\frac{1}{2}\left[l \ln \sigma_{z}^{2}+\ln |\mathbf{R}|\right] .
$$

Numerical optimization techniques are required for the optimization of (12) to estimate the unknown hyperparameters $\boldsymbol{\theta}$. Subsequently, $\mathbf{R}$ is kept as a constant matrix during predictions, unless new design points are added to the training data set. Once the metamodel has been constructed, various statistics of the output can be computed in the postprocessing phase. We now discuss the application of the surrogate model to identify the relative importance of each input variable.

\section{B. Main Effects}

It can be seen from (6) and (7) that the Bayesian model does not explicitly reveal the input-output relationships in a readily interpretable way. Consequently, this predictor is not suitable for explaining the functional relationship between the covariates and the response. In order to identify this relationship, the effect of each input needs to be isolated from the others. The response can be decomposed into main effects for each input and this main effect of the $i$ th input variable which can be obtained by integrating out the other factors is defined as follows:

$$
\gamma_{i}\left(x_{i}\right)=\frac{1}{V} \int Y(\mathbf{x}) \prod_{h \neq i} d x_{h}
$$

where $V$ is the volume of the design space over which the integration is carried out. The above integral can be numerically computed by approximating it by a sum over a grid of $m$ points $x_{h}{ }^{(1)}, \ldots, x_{h}{ }^{(m)}$. These points can be generated using Monte Carlo or Latin Hypercube sampling (LHS) techniques [11]. Note that since the Bayesian surrogate also provides the posterior variance, error bars on the main effects can be readily computed. Similarly, the effect of two or more covariates (joint effects) can be investigated by integrating out all the other covariates or by fixing the other covariates at some values [12]. The graphical plots of these effects can potentially provide useful insights into the input-output relationship.

\section{Governing Equations, Numerical Method, AND BOUNDARY CONDITIONS}

The companion paper [8] discussed the details of the exact equations governing the flow, the numerical method used to solve this problem along with the respective boundary conditions. More specific details can also be found in [15] and [16]. However, some of the details are outlined here to aid the reader. The geometry of a systemic arterial vessel generally tapers along its length. The radius of the vessel follows an exponential curve of the form

$$
r(x)=r_{u} \exp (k x)
$$

where $r_{u}$ (units- $\mathrm{cm}$ ) denotes the mean upstream cross section of the vessel, $k$ (units- $\mathrm{cm}^{-1}$ ) signifies the tapering factor and the length, $x$ (units $-\mathrm{cm}$ ), varies between 0 and $L$, where $L$ (units- $\mathrm{cm}$ ) is the length of a vessel. The upstream cross section of the artery is greater than the downstream cross section and they are related to each other according to the tapering factor

$$
k=\frac{1}{L} \log \left(\frac{r_{d}}{r_{u}}\right)
$$

where $r_{d}$ (units- $\mathrm{cm}$ ) denotes the downstream cross-section of the vessel. From the above equations, the radius of the vessel becomes

$$
r_{o}(x)=r_{u}\left(\frac{r_{d}}{r_{u}}\right)^{x / L}
$$

where $r_{o}(x)$ (units- $\mathrm{cm}$ ) is the radius of the vessel at zero transmural pressure $\left(p=p_{o}\right)$ (units- $\mathrm{mm} . \mathrm{Hg}$ ). The elastic proper- 
ties of the vessel can be estimated from the Young's modulus, $E$ (units $-\mathrm{g} \cdot \mathrm{cm}^{-1} \cdot \mathrm{s}^{-2}$ ), the wall thickness, $h$ (units- $\mathrm{cm}$ ), and the radius of the vessel, $r_{o}$. Empirically, Olufsen [15] has estimated a relation between them which is as follows:

$$
\frac{E h}{r_{o}}=k_{1} \exp \left(k_{2} r_{o}\right)+k_{3} \text {. }
$$

The empirical estimates of the constants $k_{1}, k_{2}$ and $k_{3}$ are taken as $2 \times 10^{7} \mathrm{~g} \cdot \mathrm{cm}^{-1} \cdot \mathrm{s}^{-2},-22.53 \mathrm{~cm}^{-1}$, and $8.65 \times 10^{5} \mathrm{~g} \cdot \mathrm{cm}^{-1}$. $\mathrm{s}^{-2}$, respectively. Equation (17) is used with the same values for these constants for all branches including the stenosed cases of the ICA. It is also used to represent the elasticity of the applied graft, but the constants $k_{1}, k_{2}$, and $k_{3}$ are optimized for different degrees of stenosis as described in more detail below. Effectively, the material properties of the graft are allowed to vary within $25 \%$ perturbations around the mean values of the artery such that the range of possible grafts are close to mechanical compatibility with the arteries to which they could be joined.

The total system of continuity and momentum equations can be written as

$$
\begin{aligned}
& \frac{\partial}{\partial t}\left(\begin{array}{c}
A \\
q
\end{array}\right)+\frac{\partial}{\partial x}\left(\begin{array}{c}
q \\
\left.\frac{q^{2}}{A}+\frac{f}{\rho} \sqrt{A_{o} A}\right) \\
=\left(-\frac{2 \pi \nu r q}{\delta A}+\frac{1}{\rho}\left(2 \sqrt{A}\left(\sqrt{\pi} f+\sqrt{A_{o}} \frac{d f}{d r_{o}}\right)-A \frac{d f}{d r_{o}}\right) \frac{d r_{o}}{d x}\right)
\end{array}\right.
\end{aligned}
$$

where $A$ (units- $\mathrm{cm}^{2}$ ) is the cross-sectional area, $q$ is the flow (units- $\mathrm{cm}^{3} \cdot \mathrm{s}^{-1}$ ), $f\left(r_{o}\right)=4 E h / 3 r_{o}$ (units- $\mathrm{g} \cdot \mathrm{cm}^{-1} \cdot \mathrm{s}^{-2}$ ), $A_{o}$ (units- $\mathrm{cm}^{2}$ ) is the cross-sectional area at zero transmural pressure, $\nu$ (units- $\mathrm{cm}^{2} \cdot \mathrm{s}^{-1}$ ) is the kinematic viscosity, $\delta$ (units- $\mathrm{cm}$ ) is the boundary layer thickness, $\rho$ (units- $\mathrm{g} \cdot \mathrm{cm}^{-3}$ ) is the density of blood, and $d f / d r_{o}=(4 / 3) k_{1} k_{2} \exp \left(k_{2} r_{o}\right)$ with units of $\mathrm{g} \cdot \mathrm{cm}^{-2} \cdot \mathrm{s}^{-2}$. Using (17), the pressure (units- $-\mathrm{mm} . \mathrm{Hg}$ ) is related in the state equation as

$$
p(x, t)-p_{o}=\frac{4}{3} \frac{E h}{r_{o}}\left(1-\sqrt{\frac{A_{o}}{A}}\right)
$$

where $p_{o}$ is the zero transmural pressure or the diastolic pressure.

Furthermore, a Richtmyer's two-step version [17] of the LaxWendroff explicit scheme [18] is used to solve the equations governing the flow. As an input flow condition, a characteristic pulse at the inlet of the common carotid artery (CCA) is used [19]. At the bifurcation, the flow, area, and pressure are balanced at the two time steps, giving rise to a set of 18 nonlinear equations that are solved using Newton's method by suitably evaluating the Jacobian. For the outflow boundary condition at the outlets of the ICA and external carotid artery (ECA), a three-element Windkessel model is used [20].

\section{RESULTS AND DISCUSSION}

\section{A. Identification of Key Geometric Parameters Influencing Haemodynamics}

This subsection presents preliminary studies conducted to identify key geometric parameters that influence PVF and $p_{m}^{*}$.
The geometry of the one-dimensional human carotid bifurcation as defined in [8] is used for the analysis presented here. The stenosis in the ICA is modeled using the Hicks-Henne bump function [21] which is defined as

$$
r_{h h}(x)=x_{1} \sin \left(\pi x^{\log 0.5 / \log x_{2}}\right)^{x_{3}} ; \text { for } 0 \leqslant x \leqslant 1
$$

where $r_{h h}(x)$ controls the radius of the vessel, $x_{1}$ is the maximum bump magnitude, $x_{2}$ is the location of the maximum point of the bump, and $x_{3}$ controls the width of the bump. With reference to the radius of the arterial vessel $(r(x))$ defined in [8], the effective radius of the vessel becomes

$$
r_{e}(x)=r(x)-r_{h h}(x) .
$$

In accordance with this definition, the percentage of stenosis can be evaluated as

$$
\%_{\text {stenosis }}=\left(1-\frac{r_{e}^{2}(x)}{r^{2}(x)}\right) \times 100 .
$$

A number of other stenosis models have been employed elsewhere, some of which can be found in the review article by Berger and Lou [22]. The closest example to the one considered here, employs a Gaussian shape to define the stenosis [23]. Also, early in vitro experiments used locally constricted models to investigate the effect of stenosis shape and size on flow characteristics [24]-[26]. In particular, Seeley and Young [26] experimentally considered a range of blunt-ended plugs to derive an expression for pressure drop that was then adjusted for a more realistic severe constriction. While useful results have been obtained from these other stenosis models, the real attraction of using the Hicks-Henne representation lies in its ability to generate a wide range of stenosis shapes using only three variables; thus, facilitating an efficient procedure for exploring the effect of shape change on haemodynamics.

The ranges of variation of $x_{1}, x_{2}, x_{3}$ and other geometry variables are shown in Table I. The geometry of the CCA is defined using $\mathrm{x}_{4}, \mathrm{x}_{5}$, and $\mathrm{x}_{6}$ denoting the radius of the upstream section, radius of the downstream section, and the length, respectively. For the ICA, $x_{7}$ denotes the radius of the upstream section, $\mathrm{x}_{8}$ is the radius of the downstream section, and $\mathrm{x}_{9}$ signifies the length. Similarly, for the ECA, $\mathrm{x}_{10}$ signifies the radius of the upstream section, $\mathrm{x}_{11}$ is the radius of the downstream section, and $\mathrm{x}_{12}$ denotes the length. By varying the parameters listed in Table I, 500 geometries (or training data) are created using an LHS technique [11] and parametric analysis is conducted to understand the behavior of PVF and $p_{m}^{*}$ in the ICA. Numerical simulations are performed on all the cases considered for the training data and the values of the pressure based metrics are extracted at the end of four cycles for each geometry in order to construct two surrogate models with PVF and $p_{m}^{*}$ as their outputs, respectively.

Main effect plots generated by integrating out the other covariates are shown in Fig. 2 for the case with PVF as the output. It can be clearly seen from the figure that the main effect plots of the variables $\mathrm{x}_{1}, \mathrm{x}_{2}, \mathrm{x}_{3}, \mathrm{x}_{7}$, and $\mathrm{x}_{8}$ are nonlinear while the plots of other variables are flat; as depicted for variable $x_{9}$. We 
TABLE I

VARIAbles Taken for the ANalyses to IDENTIFy the Key GeOMEtric Parameters That Influence Pressure Variation Factor

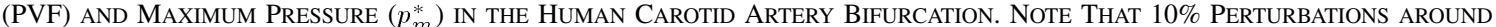
the Mean Value Are Taken for Parameters $\mathrm{x}_{i}(\mathrm{i}=4,5, \ldots, 12)$ IN This Study. All the Locations are Shown IN Fig. 4. The Mean Values Considered for This Study Are the Same as Those TaKen in the Companion PaPer [8]

\begin{tabular}{||c|c|c|c|c|c|c||}
\hline Location & Region & Description & Parameter & Mean & Lower bound & Upper bound \\
\hline 1 & Hicks-Henne & Maximum bump magnitude & $\mathrm{x}_{1}$ & - & $0 \mathrm{~cm}$ & $0.121 \mathrm{~cm}$ \\
2 & & Location of maximum bump & $\mathrm{x}_{2}$ & - & 0.2 & 0.8 \\
3 & & Width of the bump & $\mathrm{x}_{3}$ & - & $4.4 \mathrm{~cm}$ & $13.2 \mathrm{~cm}$ \\
\hline 4 & Common carotid & Upstream cross-section & $\mathrm{x}_{4}$ & $0.37 \mathrm{~cm}$ & $0.333 \mathrm{~cm}$ & $0.407 \mathrm{~cm}$ \\
5 & & Downstream cross-section & $\mathrm{x}_{5}$ & $0.37 \mathrm{~cm}$ & $0.333 \mathrm{~cm}$ & $0.407 \mathrm{~cm}$ \\
6 & & Length & $\mathrm{x}_{6}$ & $20.8 \mathrm{~cm}$ & $18.72 \mathrm{~cm}$ & $22.88 \mathrm{~cm}$ \\
\hline 7 & Internal carotid & Upstream cross-section & $\mathrm{x}_{7}$ & $0.177 \mathrm{~cm}$ & $0.1593 \mathrm{~cm}$ & $0.1947 \mathrm{~cm}$ \\
8 & & Downstream cross-section & $\mathrm{x}_{8}$ & $0.177 \mathrm{~cm}$ & $0.1593 \mathrm{~cm}$ & $0.1947 \mathrm{~cm}$ \\
9 & & Length & $\mathrm{x}_{9}$ & $17.6 \mathrm{~cm}$ & $15.84 \mathrm{~cm}$ & $19.36 \mathrm{~cm}$ \\
\hline 10 & External carotid & Upstream cross-section & $\mathrm{x}_{10}$ & $0.177 \mathrm{~cm}$ & $0.1593 \mathrm{~cm}$ & $0.1947 \mathrm{~cm}$ \\
11 & & Downstream cross-section & $\mathrm{x}_{11}$ & $0.177 \mathrm{~cm}$ & $0.1593 \mathrm{~cm}$ & $0.1947 \mathrm{~cm}$ \\
12 & & Length & $\mathrm{x}_{12}$ & $17.7 \mathrm{~cm}$ & $15.93 \mathrm{~cm}$ & $19.47 \mathrm{~cm}$ \\
\hline
\end{tabular}
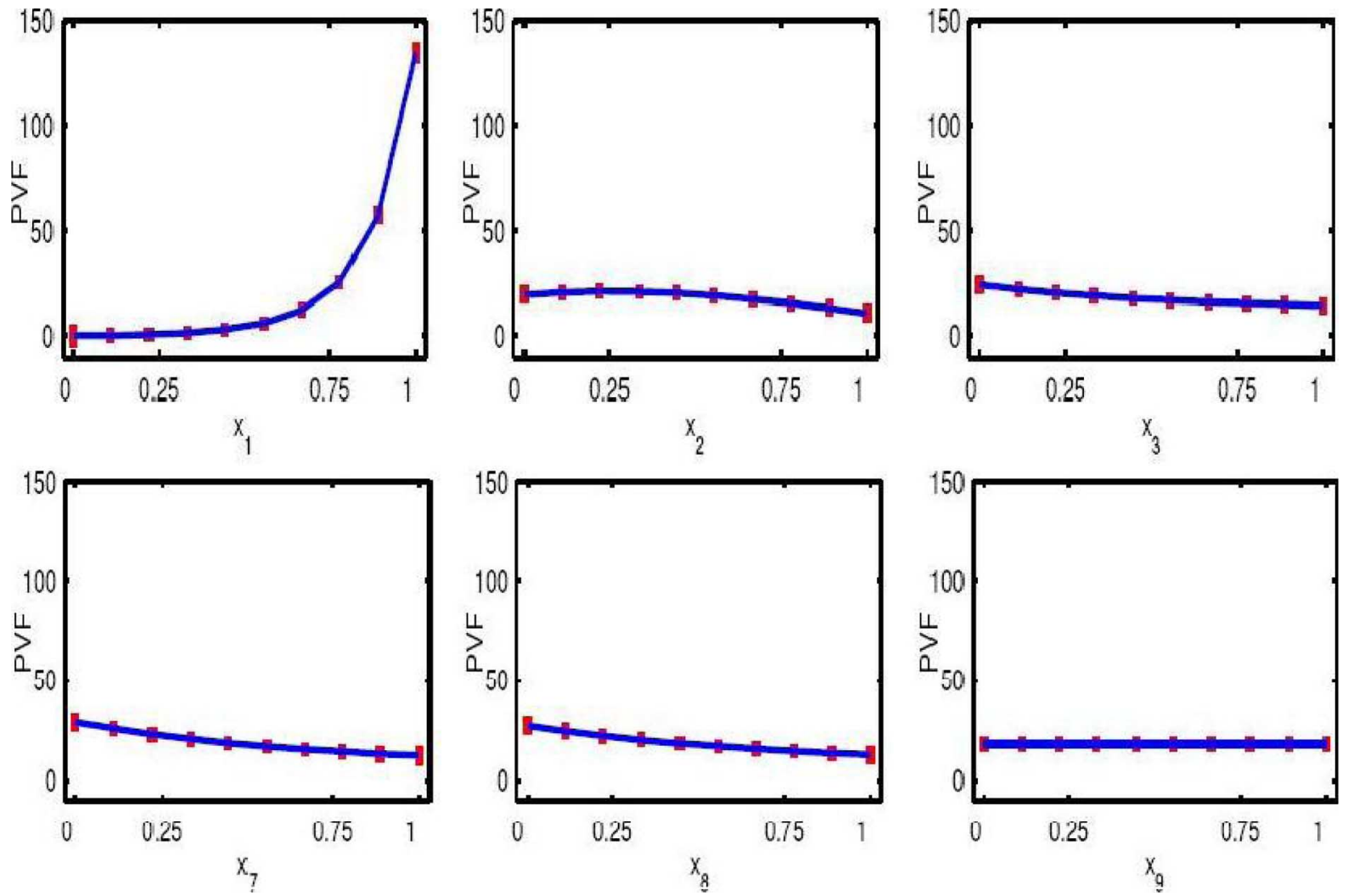

Fig. 2. Main effect plots for $x_{1}$ (maximum bump magnitude), $x_{2}$ (location of maximum bump), $x_{3}$ (width of the bump), $x_{7}$ (ICA inlet radius), $x_{8}$ (ICA outlet radius), and $x_{9}$ (ICA length) when nondimensional pressure variation factor (PVF) is the output. The middle line is the estimated effect and the error bars are $\pm 95 \%$ confidence limits based on the standard error. On the $\mathrm{x}$-axis of each subplot, all the variables are normalized using their bounds.

can see that the degree of nonlinearity of $x_{1}$ is more than that of the other variables. Hence, it can be concluded that the percentage of stenosis influences PVF more than any other parameter. Since the pressure increment is caused due to the presence of the stenosis in the ICA, it is not surprising to observe that the geometric parameters that define the stenosis shape should directly affect PVF. Indeed, it can be seen that the other geometric parameters governing the stenosis $\left(x_{2}\right.$ and $\left.x_{3}\right)$ seem to have a significant effect on PVF. Additionally, the other variables that have a noticeable effect are the inlet and outlet radii $\left(x_{7}\right.$ and $x_{8}$, respectively) of the ICA. The parameters governing the geometry of the CCA $\left(\mathrm{x}_{4}, \mathrm{x}_{5}\right.$, and $\left.\mathrm{x}_{6}\right)$ and the ECA $\left(\mathrm{x}_{10}, \mathrm{x}_{11}\right.$, and $\mathrm{x}_{12}$ ) appear to have little affect on the PVF in the ICA.
In contrast to the global pressure variation, further insight is possible by considering the local influences of geometric variables on values of maximum pressure. To do this, $p_{m}^{*}$ is extracted for each simulation and another surrogate model is constructed with $p_{m}^{*}$ as the objective. Fig. 3 shows the main effect plots for this case. Interestingly, only the main effect plots for the variables $x_{1}, x_{7}$, and $x_{8}$ are nonlinear while the others are flat; as shown by way of example for $x_{2}$. This suggests that the location of the peak and the extent of the stenosis have negligible effects locally on the pressure distribution when compared to their global effect [8]. Fig. 3 re-enforces the inference made using Fig. 2 that the percentage of stenosis is likely to be the key geometric parameter influencing the pressure distribution in the 
TABLE II

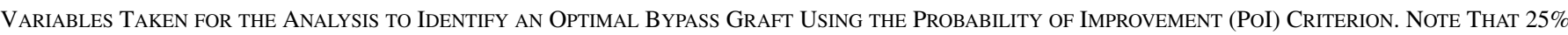
Perturbations Around the Mean Value are Taken for all the Parameters in This Study. All Locations are Shown in Fig. 4

\begin{tabular}{||c|c|c|c|c|c||}
\hline Location & Description & Parameter & Mean & Lower bound & Upper bound \\
\hline 13 & Upstream cross-section & $\mathrm{x}_{13}$ & $0.177 \mathrm{~cm}$ & $0.13275 \mathrm{~cm}$ & $0.22125 \mathrm{~cm}$ \\
14 & Downstream cross-section & $\mathrm{x}_{14}$ & $0.177 \mathrm{~cm}$ & $0.13275 \mathrm{~cm}$ & $0.22125 \mathrm{~cm}$ \\
15 & Length & $\mathrm{x}_{15}$ & $9.6 \mathrm{~cm}$ & $7.2 \mathrm{~cm}$ & $12.0 \mathrm{~cm}$ \\
\hline & Material properties & $\mathrm{k}_{1}$ & $2 \times 10^{7} \mathrm{~g} \cdot \mathrm{cm}^{-1} \cdot \mathrm{s}^{-2}$ & $1.5 \times 10^{7} \mathrm{~g} \cdot \mathrm{cm}^{-1} \cdot \mathrm{s}^{-2}$ & $2.5 \times 10^{7} \mathrm{~g} \cdot \mathrm{cm}-1$ \\
& & $\mathrm{k}_{2}$ & $-22.53 \mathrm{~cm}^{-1}$ & $-28.1625 \mathrm{~cm}^{-1}$ & $-16.8975 \mathrm{~cm}$ \\
& & $\mathrm{k}_{3}$ & $8.65 \times 10^{5} \mathrm{~g} \cdot \mathrm{cm}^{-1} \cdot \mathrm{s}^{-2}$ & $6.4875 \times 10^{5} \mathrm{~g} \cdot \mathrm{cm}^{-1} \cdot \mathrm{s}^{-2}$ & $1.08125 \times 10^{6} \mathrm{~g} \cdot \mathrm{cm}^{-1} \cdot \mathrm{s}^{-2}$ \\
\hline
\end{tabular}
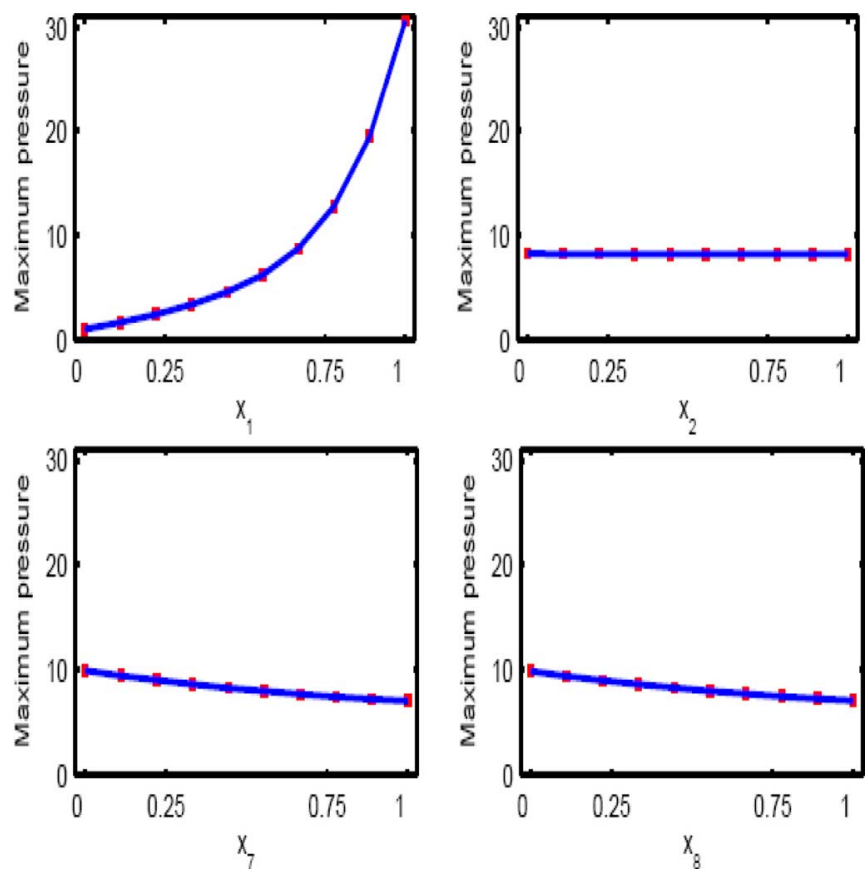

Fig. 3. Main effect plots for $x_{1}$ (maximum bump magnitude), $x_{2}$ (location of maximum bump), $x_{7}$ (ICA inlet radius), and $x_{8}$ (outlet ICA radius) when nondimensional maximum pressure $\left(p_{m}^{*}\right)$ is the output. The middle line is the estimated effect and the error bars are $\pm 95 \%$ confidence limits based on the standard error. On the $\mathrm{x}$-axis of each subplot, all the variables are normalized using their bounds.

human carotid bifurcation. As observed for the previous metric, the parameters defining the CCA and the ECA $\left(\mathrm{x}_{4}, \mathrm{x}_{5}, \mathrm{x}_{6}, \mathrm{x}_{10}\right.$, $\mathrm{x}_{11}$, and $\mathrm{x}_{12}$ ) have least influence on $p_{m}^{*}$.

It should also be observed in Fig. 3 that the values of $p_{m}^{*}$ are unexpectedly high. Scrutiny of a number of the stenosis solutions reveals that the exponential relationship between the Young's modulus $E$ and the radius at zero transmural pressure $r_{0}$ generates increasingly large values of $E h / r_{0}$ as the radius decreases through the stenosed regions. The associated pressure rise predicted by (19) causes the vessel to expand, and this further increases the pressure due to the area ratio term in (19). While (17) and (19) have been recently used elsewhere [27], these results suggest that an alternative representation of elasticity of the vessel should be used through the constrictions. Notwithstanding the need to further investigate this shortcoming, the method described so far is now applied to the design of an optimal graft, anastomosed to the bifurcation.

\section{B. Optimal Graft Design}

In this section, we illustrate the application of Bayesian surrogate modeling to predict a graft configuration (cf. Fig. 4) which

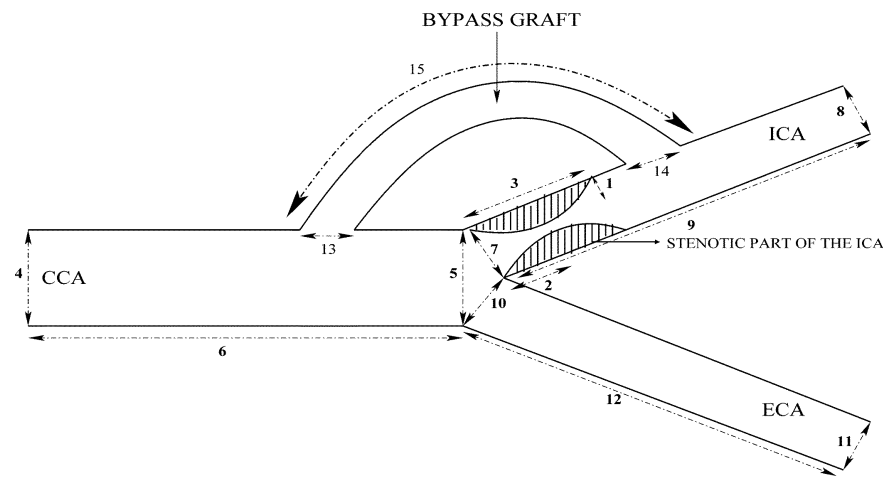

Fig. 4. Schematic of the one-dimensional human carotid artery bifurcation with a stenosis at the ICA along with the bypass graft.

has minimum PVF in the human carotid bifurcation. A straightforward way to do this would be to directly minimize the PVF predicted by the surrogate model—by performing a search procedure on the surrogate predictor-as a function of the variables governing the geometric and material properties of the graft. Alternatively, the error bar predicted by the surrogate can be employed to maximize the probability of improvement (PoI) criterion

$$
\text { Maximize : } \mathrm{P}\left[Y(\mathbf{x})<y^{-}\right]
$$

where $y^{-}$denotes the lowest value of PVF among all the geometries in the training data set used to construct the surrogate model. Note that since the surrogate prediction $Y(\mathbf{x})$ is a Gaussian random field, the PoI can be computed exactly.

As a test case, a geometry having $80 \%$ stenosis is considered with $\mathrm{x}_{2}$ fixed at $0.5, \mathrm{x}_{3}$ fixed at $4.4 \mathrm{~cm}$, and the variables $\left\{\mathrm{x}_{4}, \mathrm{x}_{5}, \ldots, \mathrm{x}_{12}\right\}$ fixed at their mean values (cf. Table I). The six parameters which govern the geometric and material properties of the bypass graft (cf. Table II) are varied to create training data for constructing the surrogate model. Note that $x_{13}$ controls the upstream cross section, $x_{14}$ controls the downstream cross-section, and $x_{15}$ controls the length of the bypass graft, respectively. The material properties of the graft are governed by (17) such that the constants $\mathrm{k}_{1}, \mathrm{k}_{2}$, and $\mathrm{k}_{3}$ are allowed to vary in the search for an optimum. Intrinsically, it is assumed that the material properties of the bypass graft closely match to that of a systemic elastic artery. Furthermore, for a tapering graft, it can be seen from (17) that the elasticity increases along its length.

Fig. 5 shows the main effects plots generated for the variables $\mathrm{x}_{13}, \mathrm{x}_{14}, \mathrm{x}_{15}, \mathrm{k}_{1}, \mathrm{k}_{2}$, and $\mathrm{k}_{3}$. It can be seen that the degree of nonlinearity of the variable $k_{2}$ is more than that of any other variable. In addition, it is clearly evident that the influence of the variables $k_{1}$ and $k_{3}$ on PVF is more considerable than $\mathrm{x}_{13}$, 

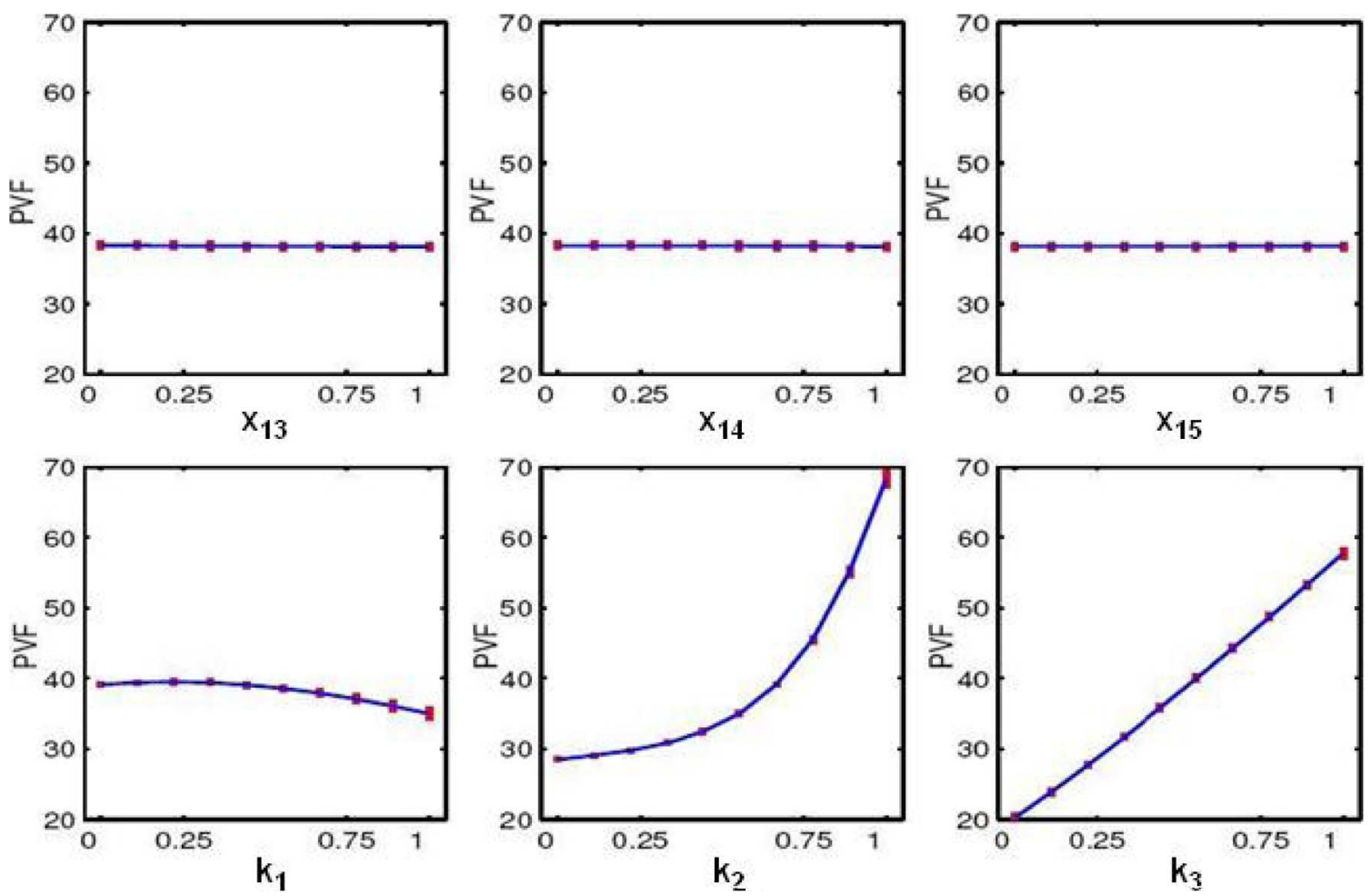

Fig. 5. Main effect plots for the case with $80 \%$ stenosis at the ICA. $\left(x_{13}, x_{14}\right.$, and $x_{15}$ are, respectively, the graft inlet radius, outlet radius, and length. $k_{1}, k_{2}$ and $k_{3}$ denote the constants defining the material properties of the graft as defined in (17)). The middle line is the estimated effect and the error bars are $\pm 95 \%$ confidence limits based on the standard error. Nondimenional PVF is shown on the $\mathrm{y}$-axis of each subplot. On the $\mathrm{x}$-axis of each subplot, all the variables are normalized using their bounds.

$\mathrm{x}_{14}$, and $\mathrm{x}_{15}$. This observation may directly suggest that the material properties of the bypass graft have a greater influence on the pressure distribution than the geometric properties in this one-dimensional study. Although this statement contradicts the general consensus that the shape of the arteries is the most important factor influencing the haemodynamics [28], quantifying the overall effect of the pressure increment in terms of a scalar metric for this one-dimensional study highlights the importance of the material properties as well.

The objective of this optimization study is to predict a bypass graft configuration which produces a low value of PVF in the original bifurcation. The motivation for minimizing PVF arises from the fact that this metric quantifies the extent of pressure build up relative to normal behavior in the entire vessel. Although changes in flow leaving the ICA and ECA can have an effect on the graft configuration, the main concern is to obtain an optimal design with respect to variations in pressure. The underlying assumption is that the most suitable configuration for the bypass is the one which has minimum PVF. Hence, by minimizing the PoI criterion on the training data set, better designs of the bypass graft are obtained, compared to the initial configurations. For the case with $80 \%$ stenosis, a graft configuration is predicted which has a lower value of PVF compared to the values in the training data set. Note that the minimum value of PVF in the training data set is 12.5454 , while the PVF value predicted by the surrogate model for the optimal graft configuration was found to be 12.1367. Numerical simulations were later performed on this optimal case and it was found that the CFD predicted value of PVF was 12.1721. Thus, the configu- ration presented in Table III for the case with $80 \%$ stenosis in the ICA denotes a design which is better than any of the cases belonging to the training data. Note that this criterion can also be used for updating the surrogate model to find a better optima. The new point obtained can be appended to the initial training data set and the criterion can be applied in an iterative fashion to find optimal bypass graft configurations. Alternative strategies for finding an optimal design can be found in [29]. Furthermore, the capability of using the PoI criterion to predict better graft designs has been harvested for cases with different degrees of stenosis. Table III shows optimal graft configurations predicted using the PoI criterion for cases with $70 \%, 75 \%, 80 \%, 85 \%$, and $90 \%$ stenosis, respectively, all for a single update. In each stenosis case, the values $x_{4}$ to $x_{12}$ defining the shape of the underlying bifurcation are held fixed at their mean values.

\section{CONCLUSION}

In this paper, a Bayesian surrogate modeling technique has been presented for predicting optimal arterial bypass graft configurations for a model of the human carotid bifurcation with stenosis in the internal carotid artery. A one-dimensional time dependent Navier-Stokes solver incorporating fluid-wall interactions was implemented for studying blood flow through this model. A design of experiments technique was used to create geometries for a parametric study in order to identify key geometric parameters that influence pressure variation factor and maximum pressure in the human carotid bifurcation. A Bayesian surrogate model was constructed for statistically analyzing how each geometric parameter influences the pressure 
TABLE III

Optimal Bypass Graft Configurations Obtained Using the Probability of IMProvement (PoI) Criterion For Five Test CASES

\begin{tabular}{|c|c|c|c|}
\hline & Description & Parameter & Optimal value \\
\hline \multirow{6}{*}{$\begin{array}{c}\text { CASE - 1 } \\
70 \% \text { stenosis in the ICA }\end{array}$} & Geometry & $\mathrm{x}_{13}$ & $0.1565 \mathrm{~cm}$ \\
\hline & & $\mathrm{x}_{14}$ & $0.2205 \mathrm{~cm}$ \\
\hline & & $\mathrm{X}_{15}$ & $12.383 \mathrm{~cm}$ \\
\hline & Material properties & $\mathrm{k}_{1}$ & $2.4097 \times 10^{7}$ g.cm ${ }^{-1} \cdot \mathrm{s}^{-2}$ \\
\hline & & $\mathrm{k}_{2}$ & $-21.229 \mathrm{~cm}^{-1}$ \\
\hline & & $\mathrm{k}_{3}$ & $6.6093 \times 10^{5} \mathrm{~g} \cdot \mathrm{cm}^{-1} \cdot \mathrm{s}^{-2}$ \\
\hline \multirow{6}{*}{$\begin{array}{c}\text { CASE - 2 } \\
75 \% \text { stenosis in the ICA }\end{array}$} & Geometry & $\mathrm{x}_{13}$ & $0.1618 \mathrm{~cm}$ \\
\hline & & $\mathrm{x}_{14}$ & $0.1502 \mathrm{~cm}$ \\
\hline & & $\mathrm{x}_{15}$ & $8.7182 \mathrm{~cm}$ \\
\hline & Material properties & $\mathrm{k}_{1}$ & $2.4596 \times 10^{7}$ g.cm ${ }^{-1} \cdot \mathrm{s}^{-2}$ \\
\hline & & $\mathrm{k}_{2}$ & $-22.365 \mathrm{~cm}^{-1}$ \\
\hline & & $\mathrm{k}_{3}$ & $6.6510 \times 10^{5}$ g.cm ${ }^{-1} \cdot \mathrm{s}^{-2}$ \\
\hline \multirow{6}{*}{$\begin{array}{c}\text { CASE - 3 } \\
80 \% \text { stenosis in the ICA }\end{array}$} & Geometry & $\mathrm{X}_{13}$ & $0.2048 \mathrm{~cm}$ \\
\hline & & $\mathrm{x}_{14}$ & $0.1662 \mathrm{~cm}$ \\
\hline & & $\mathrm{x}_{15}$ & $10.049 \mathrm{~cm}$ \\
\hline & Material properties & $\mathrm{k}_{1}$ & $2.4599 \times 10^{7}$ g.cm ${ }^{-1} \cdot \mathrm{s}^{-2}$ \\
\hline & & $\mathrm{k}_{2}$ & $-23.731 \mathrm{~cm}^{-1}$ \\
\hline & & $\mathrm{k}_{3}$ & $6.7666 \times 10^{5}$ g.cm ${ }^{-1} \cdot \mathrm{s}^{-2}$ \\
\hline \multirow{6}{*}{$\begin{array}{c}\text { CASE - } 4 \\
85 \% \text { stenosis in the ICA }\end{array}$} & Geometry & $\mathrm{x}_{13}$ & $0.1627 \mathrm{~cm}$ \\
\hline & & $\mathrm{X}_{14}$ & $0.1466 \mathrm{~cm}$ \\
\hline & & $\mathrm{x}_{15}$ & $11.662 \mathrm{~cm}$ \\
\hline & Material properties & $\mathrm{k}_{1}$ & $2.4780 \times 10^{7}$ g.cm ${ }^{-1} \cdot \mathrm{s}^{-2}$ \\
\hline & & $\mathrm{k}_{2}$ & $-26.914 \mathrm{~cm}^{-1}$ \\
\hline & & $\mathrm{k}_{3}$ & $6.7257 \times 10^{5} \mathrm{~g} \cdot \mathrm{cm}^{-1} \cdot \mathrm{s}^{-2}$ \\
\hline \multirow{6}{*}{$\begin{array}{c}\text { CASE - } 5 \\
90 \% \text { stenosis in the ICA }\end{array}$} & Geometry & $\overline{\mathrm{X}_{13}}$ & $0.1746 \mathrm{~cm}$ \\
\hline & & $\mathrm{x}_{14}$ & $0.1652 \mathrm{~cm}$ \\
\hline & & $\mathrm{X}_{15}$ & $11.035 \mathrm{~cm}$ \\
\hline & Material properties & $\mathrm{k}_{1}$ & $2.4697 \times 10^{7}$ g.cm ${ }^{-1} \cdot \mathrm{s}^{-2}$ \\
\hline & & $\mathrm{k}_{2}$ & $-27.200 \mathrm{~cm}^{-1}$ \\
\hline & & $\mathrm{k}_{3}$ & $6.5184 \times 10^{5}$ g.cm ${ }^{-1} \cdot \mathrm{s}^{-2}$ \\
\hline
\end{tabular}

variation factor and maximum pressure. Percentage of stenosis was found to influence these metrics more than any other variable. Furthermore, a probability of improvement criterion was used to predict bypass grafts having optimal geometric and material properties, for a given degree of stenosis.

Although this work employs a one-dimensional model and other simplifications to simulate the flow in the carotid bifurcation, the research presented in this paper and in [8] has successfully demonstrated the possibility of designing optimal arterial grafts and stents using nondeterministic and deterministic techniques, respectively. Further confidence in these techniques should be provided using more accurate methods obtainable from higher dimensional models (e.g., three-dimensional CFD [30]) and/or improved representations of arterial material properties. Nonetheless, it is hoped that these techniques could also be applied to the design of a wide range of interventional devices at different locations in the human anatomy.

This optimal design strategy could be used to handle patient-specific geometric design of arterial grafts and stents. More specifically, using this paradigm, it may be possible to predict the efficacies of stenting or grafting on an individual patient. In practice, diagnostic information could be provided for a human subject with a certain degree of stenosis by first evaluating PVF and $p_{m}^{*}$ in a model of the stenosed geometry. Later, a graft or a stent could be selected only if the corresponding metrics were reduced following simulation through the reconstructed model [8]. Additionally, a representation of a patient's geometry could be appended to the training data for reconstructing the surrogate model. As a consequence, it may be possible to design patient-specific optimal grafts or stents using the Bayesian surrogate modeling technique that can then be manufactured using materials that are mechanically and biologically compatible with the arteries they are designed to treat.

\section{ACKNOWLEDGMENT}

The authors would like to thank the reviewers for their comments and suggestions. This research was performed during V. B. Kolachalama's doctoral study and he thanks the School of Engineering Sciences at the University of Southampton for providing a studentship.

\section{REFERENCES}

[1] H. J. M. Barnett, D. W. Taylor, M. Eliasziw, A. J. Fox, G. G. Ferguson, R. B. Haynes, R. N. Rankin, G. P. Clagett, V. C. Hachinski, D. L. Sackett, K. E. Thorpe, and H. E. Meldrum, "Benefit of carotid endarterectomy in patients with symptomatic moderate or severe stenosis," New England J. Med., vol. 339, no. 20, pp. 1415-1425, 1998.

[2] J. S. Yadav, M. H. Wholey, R. E. Kuntz, P. Fayad, B. T. Katzen, G. J. Mishkel, T. K. Bajwa, P. Whitlow, N. E. Strickman, M. R. Jaff, J. J. Popma, D. B. Snead, D. E. Cutlip, B. G. Firth, and K. Ouriel, "Protected carotid-artery stenting versus endarterectomy in high-risk patients," New England J. Med., vol. 351, no. 15, pp. 1493-1501, 2004.

[3] S. P. Roddy, R. C. Darling, III, K. J. Ozsvath, M. Mehta, B. B. Chang, P. S. K. Paty, P. B. Kreienberg, and D. M. Shah, "Choice of material for internal carotid artery bypass grafting: Vein or prosthetic? Analysis of 44 procedures," Cadiovascular Surg., vol. 10, pp. 540-544, 2002. 
[4] K. W. Neff, P. Horn, D. Dinter, P. Vajkoczy, P. Schmiedek, and C. Düber, "Extracranial-intracranial arterial bypass surgery improves total brain blood supply in selected symptomatic patients with unilateral internal carotid artery occlusion and insufficient collateralization," Neuroradiology, vol. 46, pp. 730-737, 2004.

[5] D. A. Vorp, D. A. Steinman, and C. R. Ethier, "Computational modeling of arterial biomechanics," Comput. Sci. Eng., vol. 3, no. 5, pp. $51-62,2001$.

[6] C. M. Su, D. Lee, R. Tran-Son-Tay, and W. Shyy, "Fluid flow structure in arterial bypass anastomosis," J. Biomech. Eng., vol. 127, pp. $611-618,2005$.

[7] S. Giordana, S. J. Sherwin, J. Peiró, D. J. Doorly, J. S. Crane, K. E. Lee, N. J. W. Cheshire, and C. G. Caro, "Local and global geometric influence on steady flow in distal anastomoses of peripheral bypass grafts," J. Biomech. Eng., vol. 127, pp. 1087-1098, 2005.

[8] V. B. Kolachalama, N. W. Bressloff, P. B. Nair, and C. P. Shearman, "Predictive haemodynamics in a one-dimensional human carotid artery bifurcation. Part I: Application to stent design," IEEE Trans. Biomed. Eng., vol. 54, no. 5, pp. 802-812, May 2007.

[9] V. B. Kolachalama, "Predictive haemodynamics of the human carotid artery," Ph.D. dissertation, Univ. of Southampton, Southampton, U.K., 2006.

[10] V. B. Kolachalama, N. W. Bressloff, and P. B. Nair, "Multiobjective shape optimization of the human carotid artery," in Proc. 3rd MIT Conf. Comput. Fluid Solid Mech., Cambridge, MA, 2005, pp. 1310-1314.

[11] M. D. McKay, W. J. Conover, and R. J. Beckman, "A comparison of three methods for selecting values of input variables in the analysis of output from a computer code," Technometrics, vol. 21, pp. 239-245, 1979.

[12] J. Sacks, W. J. Welch, T. J. Mitchell, and H. P. Wynn, "Design and analysis of computer experiments," Statist. Sci., vol. 4, no. 4, pp. 409-435, 1989.

[13] P. B. Nair, A. Choudhury, and A. J. Keane, "A Bayesian framework for uncertainty analysis using deterministic black-box simulation codes," in Proc. 42nd AIAA/ASME/ASCE/AHS/ASC Structures, Seattle, WA, 2001, AIAA Paper No. 2001-1677.

[14] C. Currin, T. Mitchell, M. Morris, and D. Ylvisaker, "Bayesian prediction of deterministic functions with applications to the design and analysis of computer experiments," J. Amer. Statist. Assoc., vol. 86, pp. 953-963, 1991.

[15] M. S. Olufsen, "Modeling the arterial system with reference to an anesthesia simulator," Ph.D. dissertation, Roskilde Univ., Roskilde, Denmark, 1998.

[16] M. S. Olufsen, C. S. Peskin, W. Y. Kim, E. M. Pederson, A. Nadim, and J. Larsen, "Numerical simulation and experimental validation of blood flow in arteries with structured tree outflow conditions," Ann. Biomed. Eng., vol. 28, pp. 1281-1299, 2000.

[17] R. D. Richtmyer and K. W. Morton, Difference Methods for Initial Value Problems, 2nd ed. New York: Wiley, 1967.

[18] P. D. Lax and B. Wendroff, "Difference schemes for hyperbolic equations with high order of accuracy," Commun. Pure Appl. Math., vol. 17, pp. 381-398, 1964.

[19] D. W. Holdsworth, C. J. D. Norley, R. Frayne, D. A. Steinman, and B. K. Rutt, "Characterization of common carotid artery blood-flow waveforms in normal human subjects," Physiological Meas., vol. 20, pp. 219-240, 1999.
[20] N. Stergiopulos, D. F. Young, and T. R. Rogge, "Computer simulation of arterial flow with applications to arterial and aortic stenoses," $J$. Biomech., vol. 23, no. 1, pp. 1477-1488, 1992.

[21] R. Hicks and P. Henne, "Wing design by numerical optimization," $J$. Aircraft, vol. 15, no. 7, pp. 407-413, 1978.

[22] S. A. Berger and L.-D. Jou, "Flows in stenotic vessels," Annu. Rev. Fluid Mech., vol. 32, pp. 347-382, 2000.

[23] J. S. Stroud, S. A. Berger, and D. Saloner, "Influence of stenosis morphology on flow through severely stenotic vessels: Implications for plaque rupture," J. Biomech., vol. 33, no. 4, pp. 443-455, 2000.

[24] D. F. Young and F. Tsai, "Flow characteristics in models of arterial stenoses-I. Steady flow," J. Biomech., vol. 6, no. 4, pp. 395-402, 1973.

[25] D. F. Young and F. Tsai, "Flow characteristics in models of arterial stenoses-II. Unsteady flow," J. Biomech., vol. 6, no. 5, pp. 547-559, 1973.

[26] B. D. Seeley and D. F. Young, "Effect of geometry on pressure losses across models of arterial stenoses," J. Biomech., vol. 9, no. 7, pp. 439-448, 1976.

[27] B. N. Steele, J. Wan, J. P. Ku, T. J. R. Hughes, and C. A. Taylor, "In vivo validation of a one-dimensional finite-element method for predicting blood flow in cardiovascular bypass grafts," IEEE Trans. Biomed. Eng., vol. 50, pp. 649-656, 2003.

[28] B. K. Bharadvaj, R. F. Mabon, and D. P. Giddens, "Steady flow in a model of the human carotid bifurcation," J. Biomech., vol. 15, pp. 349-362, 1982.

[29] A. J. Keane and P. B. Nair, Computational Approaches to Aerospace Design. The Pursuit of Excellence. New York: Wiley, 2005.

[30] N. W. Bressloff, "Parametric geometry exploration of the human carotid artery bifurcation," J. Biomech., vol. 40, no. 11, pp. 2483-2491, 2007.

Vijaya B. Kolachalama, photograph and biography not provided at the time of publication.

Neil W. Bressloff, photograph and biography not provided at the time of publication.

Prasanth B. Nair, photograph and biography not provided at the time of publication.

Clifford P. Shearman, photograph and biography not provided at the time of publication. 